Arch. Dis. Childh., 1968, 43, 486.

\title{
Ventilating the Lungs of Newborn Infants for Prolonged Periods
}

\author{
M. E. TUNSTALL, J. I. CATER, JEAN S. THOMSON, and R. G. MITCHELL \\ From the Department of Child Health, University of Aberdeen; the Aberdeen Maternity Hospital; and \\ the Department of Anaesthetics, Aberdeen
}

The treatment of selected respiratory problems in newborn infants by intermittent positive pressure ventilation (IPPV) combined with naso-tracheal intubation is now well established as a routine procedure in Aberdeen, and it would be unfortunate if ill-founded fears prevented the wider use of this valuable technique. The method we use has been recorded elsewhere (Reid and Tunstall, 1966), but we here describe the technique in detail, based on our experience of treating some 90 infants (Table).

\section{Endotracheal Tube}

Portex ivory vinyl endotracheal tubest of sizes $2.5 \mathrm{~mm}$. and $3.0 \mathrm{~mm}$. internal diameter have proved most satisfactory. Ivory vinyl tubes provide a slippery internal surface for the passage of suction catheters. Equivalent tubes in clear plastic are more sticky, and make it more difficult to insert suction catheters to the prescribed depth.

Fig. 1 shows an ivory vinyl tube freshly withdrawn from a baby and detached from its metal connector. Notice the shape the tube has adopted from the course of the baby's airway. The tubes mould at body temperature. The small hole which has been cut near the tip of the tube (seen also in Fig. 2 and 11) is to reduce the danger of respiratory obstruction should 'bevelling' occur.

Fig. 2 shows a tube attached to the metal endotracheal tube connector immediately before insertion down the baby's nostril. The tubes are softened beforehand by boiling to facilitate attachment to the connector. The $2.5 \mathrm{~mm}$. tube is

\footnotetext{
* For the 'Personal Practice' series of articles, an individual author is ordinarily invited to express his views on a current topic. The subject chosen in this instance is one demanding a team approach, the multiple authorship here reflecting the collaboration between anaesthetists and paediatricians which has been an essential feature of the Aberdeen group.

† Portex tubing and endotracheal tubes from Portland Plastics Ltd., Hythe, Kent.
}

most commonly used. The $3 \mathrm{~mm}$. tube is used, usually for mature babies, if the leak of air past the larynx on positive pressure of $20 \mathrm{~cm}$. water appears to be excessive. The ideal size of tube for

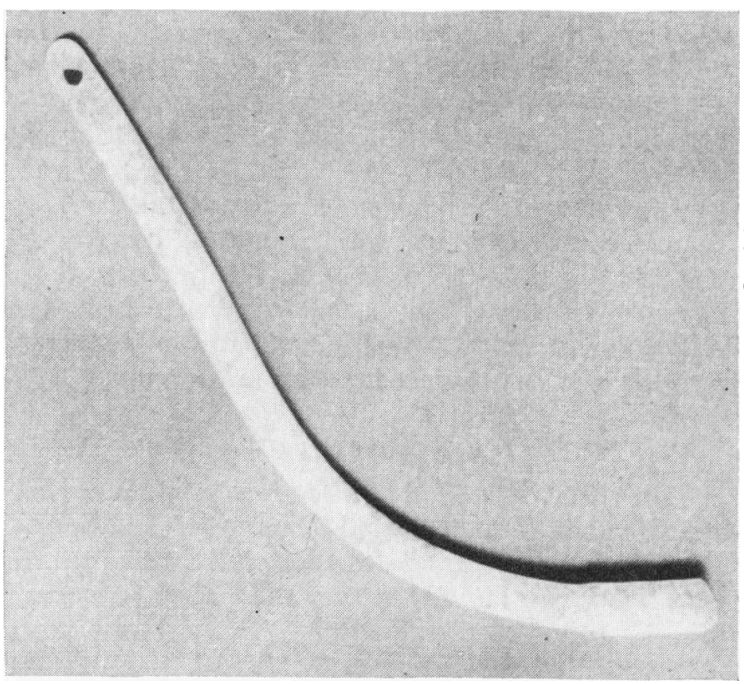

Fig. 1.-A recently used Portex ivory vinyl endotracheal tube (naso-tracheal).

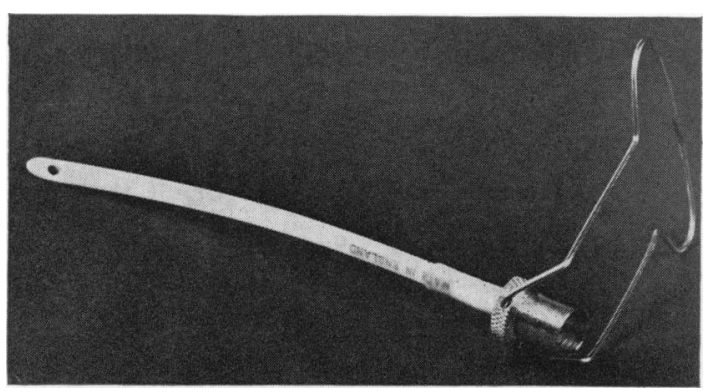

Fig. 2.-A fresh Portex ivory vinyl endotracheal tube prepared for naso-tracheal intubation. 
TABLE

Newborn Infants Treated by Prolonged Intermittent Positive Pressure Ventilation (IPPV) with Naso-tracheal Intubation

\begin{tabular}{|c|c|c|c|c|c|c|}
\hline & & & All Cases & Died & Alive & $\%$ Survival \\
\hline $\begin{array}{l}\text { Total number of infants } \\
\text { Mean body weight at birth (g.) } \\
\text { Body weight (g.) (range) } \\
\text { Mean duration of IPPV (hr.) }\end{array}$ & $\begin{array}{l}\cdots \\
\cdots \\
\cdots \\
\cdots\end{array}$ & $\begin{array}{ll}\cdots & \cdots \\
\cdots & \cdots \\
\cdots & \cdots \\
\cdots & \cdots\end{array}$ & $\begin{array}{c}90 \\
1820 \\
875-4740 \\
55 \frac{1}{2}\end{array}$ & $\begin{array}{c}57 \\
1731 \\
875-4740 \\
45\end{array}$ & $\begin{array}{c}33 \\
1971 \\
1030-2990 \\
73\end{array}$ & 37 \\
\hline 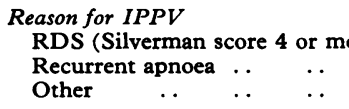 & $\begin{array}{l}\text { e an } \\
\cdots \\
\cdots\end{array}$ & \begin{tabular}{lr|}
$\mathbf{H}<\mathbf{7} \cdot 2)$ \\
$\cdots$ & $\cdots$ \\
$\cdots$ & $\cdots$
\end{tabular} & $\begin{array}{l}38 \\
35 \\
17\end{array}$ & $\begin{array}{l}22 \\
21 \\
14\end{array}$ & $\begin{array}{r}16 \\
14 \\
3\end{array}$ & $\begin{array}{l}42 \\
40 \\
18\end{array}$ \\
\hline
\end{tabular}

IPPV in babies is one that allows a slight audible air leak back past the larynx when a positive pressure of $20 \mathrm{~cm} . \mathrm{H}_{2} \mathrm{O}$ is applied.

No infant has required either re-intubation or

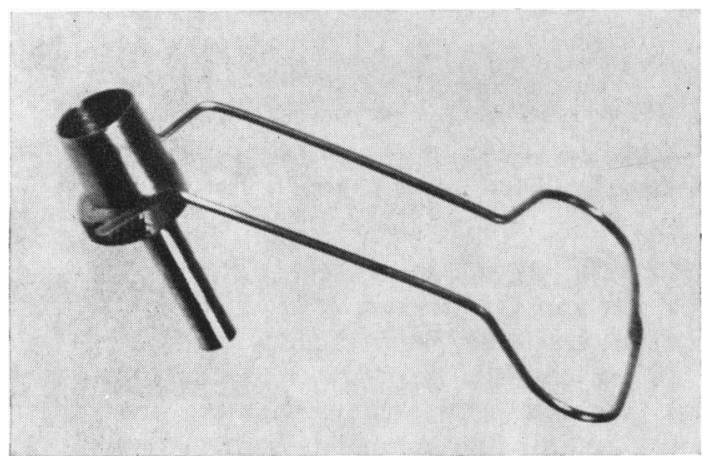

Fig. 3.-The modified new Oxford pattern endotracheal tube connector (from Anaesthesia, 21, 72-by permission of the Editor). tracheostomy for subglottic oedema or laryngeal complication, and there has been no lasting laryngeal or tracheal morbidity in any of our survivors. No cases coming to necropsy have shown damage due to prolonged intubation.

\section{Endotracheal Tube Connector}

This connector (Fig. 3 ) is a modified new Oxford pattern endotracheal tube connector*, a single item which is considered to be a major factor in the trouble-free management of neonates on IPPV. It can be seen from Fig. 4 and 5 that it provides: (1) great security of fixation of an indwelling endotracheal tube; (2) freedom to adopt the supine or lateral postures; (3) ease of disconnexion from ventilator tubing for purposes of tracheal toilet, transfer, changing posture, etc.; (4) easy access to scalp veins.

^ Longworth Scientific Instrument Co. Ltd., Abingdon, Berks.

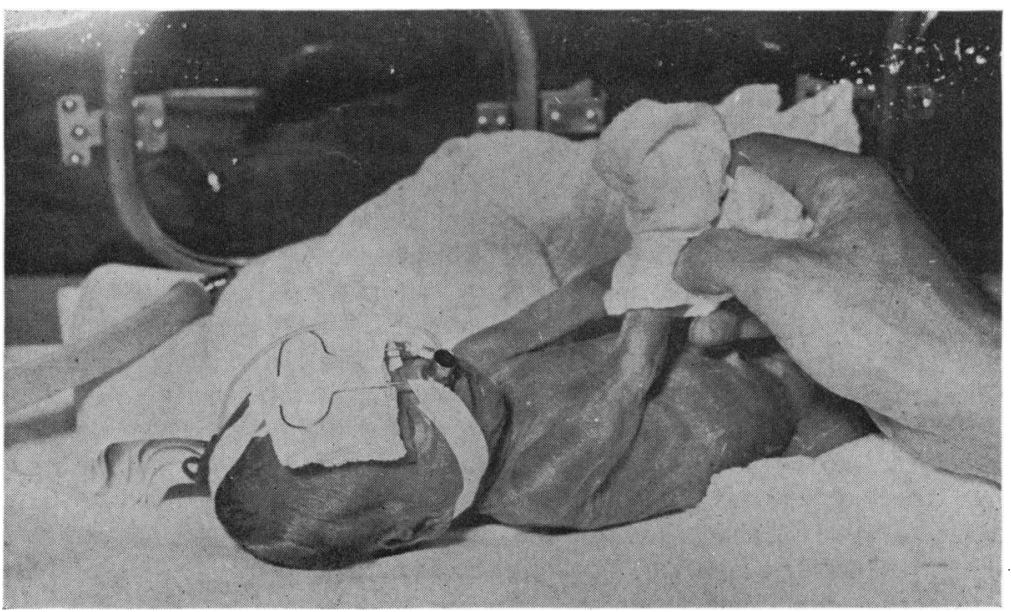

FIG. 4.-Secure naso-tracheal intubation. 


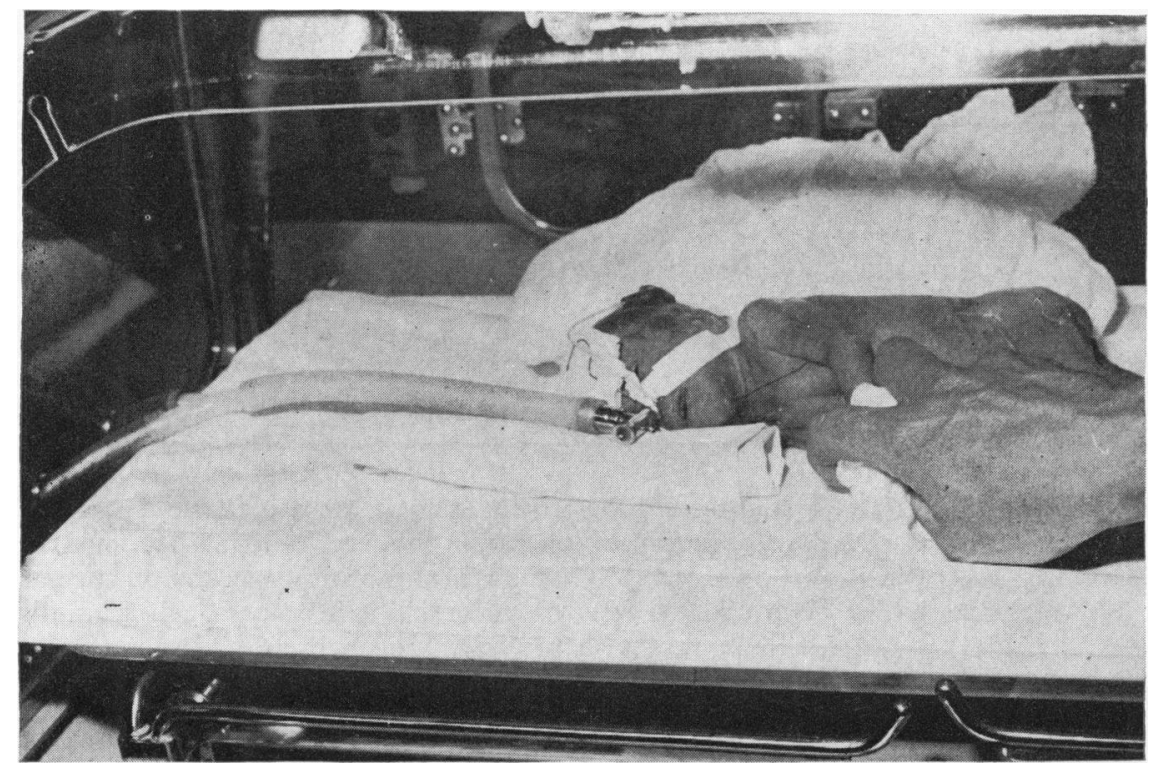

Fig. 5.-Artificial ventilation in progress. Note (1) position of the covered sandbags; (2) low position of ports for ventilator tubing; (3) in this case, the deliberately increased dead space.

\section{Airway}

An insecure airway is misery for the attendants and may be fatal to the patient. An adequately secured naso-tracheal tube with its tip 1-2 cm. above the carina is not subjected to kinking by any posture adopted by the baby's head. It is, however, important to observe the rules in Fig. 6.

If the endotracheal tube is too short the tip may spring out of the larynx when the baby's head is moved or the tip may catch anteriorly in the larynx. This latter situation may either cause obstruction directly, or the tube may then kink when the head is moved again. If the tube is a little too long, there may be ventilation of only one lung and/or intermittent obstruction caused by the bevel of the tube impinging directly against the wall of a bronchus.

The distance from the nostril to the lower part of the trachea in the neonate is judged to be $1 \frac{1}{2}$ times the distance from the nostril to the tragus of the ear, the tube being held against the infant's cheek. The tube is then cut to this length. Such a length is sometimes too long, rarely too short.

After intubation and full insertion of the connector into the nose, it should be possible to withdraw the tube at least $2 \mathrm{~cm}$. back from its fully inserted position without its coming out of the larynx. This can be checked with the laryngoscope still in place and with the laryngeal entrance in view.

After fixation of the tube, the baby's head should be gently placed in several different limits of position and breath sounds produced by artificial ventilation checked on both sides of the chest.

If the length is not right, a second tube which was prepared for use along with the first tube is now cut to the estimated correct length. If necessary, in the meantime, the baby is kept oxygenated by replacement of the oro-tracheal resuscitation tube (Tunstall and Hodges, 1961), and ventilation with oxygen is maintained manually. $X$-ray of the chest can, if necessary, be used to check the position of the tube (Fig. 7).

When fixing the connector, the two lateral straps of adhesive (zinc oxide) tape are applied first and, after slight forward traction of the skin of the cheek, are taken round the back of the neck. The mid-line head strap is tensed to give correct fixation of the connector. With correct fixation it should be possible to pull the connector half a centimetre out of the nostril but no further.

The skin of the baby is protected by the application of benzoin tincture before intubation and the application of zinc oxide tape. This tape is cut adjacent to the metal connector when it is desired to take the baby off the ventilator and remove the endotracheal tube. The tape is not peeled off the skin at this stage, for, should it be necessary for the baby to go back on the ventilator, fresh tape is placed over that existing. 


\section{THE MANAGEMENT OF INDWELLING PLASTIC NASOTRACHEAL TUBES.}

The plastic tube runs up from the trachea through the larynx, via the oropharynx and out to the nose where it is fixed onto a special metal connector in one or other nostril. This arrangement is a substitute for a tracheostomy and provides an accessible airway without a surgical operation.

It is important that the strapping holding the connector in place is never touched. If it becomes loose it should be reported at once. If the patient is on a respirator at no time must the respirator tubing be allowed to drag on or twist the connector strapped to the patient. This can be checked by confirming that when the patient connector and the respirator connector are disconnected from each other, they do not spring widely apart or twist out of line with each other. Check that the connector in the nose always points in a vertical plane straight from the centre of the forehead to the centre of the occiput as in $A$ :
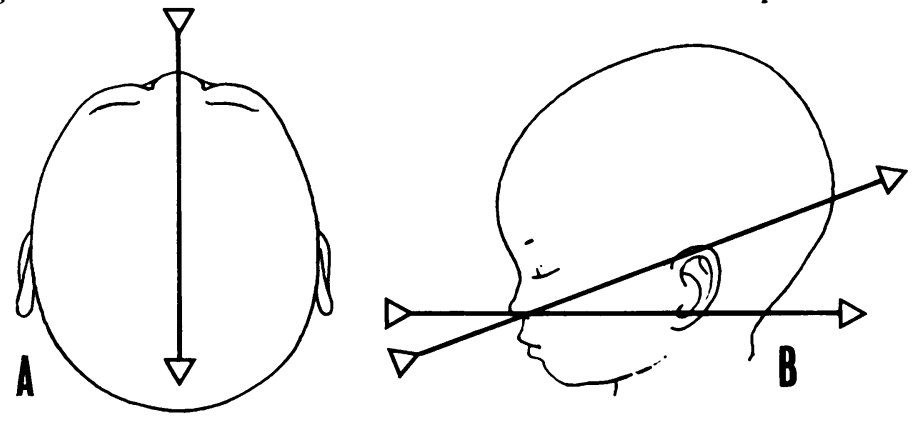

Also check that the direction of the connector does not stray away from the limit of the planes shown as in B:

FIG. 6.-Notice posted in the ventilator room.

\section{Technique of Naso-tracheal Intubation}

Visualization of the laryngeal opening with the aid of a laryngoscope should be learned from someone proficient in the art, and skill should be acquired by practice on infants who are stillborn or have died shortly after birth. A laryngoscope with the light-weight Anderson handle ${ }^{\star}$ and the Magill straight infant blade is advised.

The baby should be on a flat surface without undue extension of the head. The blade of the laryngoscope is passed so as to displace the tongue to the left, and the tip of the blade is then placed at the junction of the tongue and epiglottis and the blade lifted, using the upper gum to obtain gentle leverage. An assistant may be asked to press on the anterior aspect of the neck with the ulnar border of her hand, for this helps to swing the laryngeal opening into view. The laryngoscope should be held in the mid-line by the operator's left hand, the little finger and ulnar border of which can simultaneously be steadying the chin. The opera-

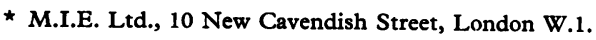

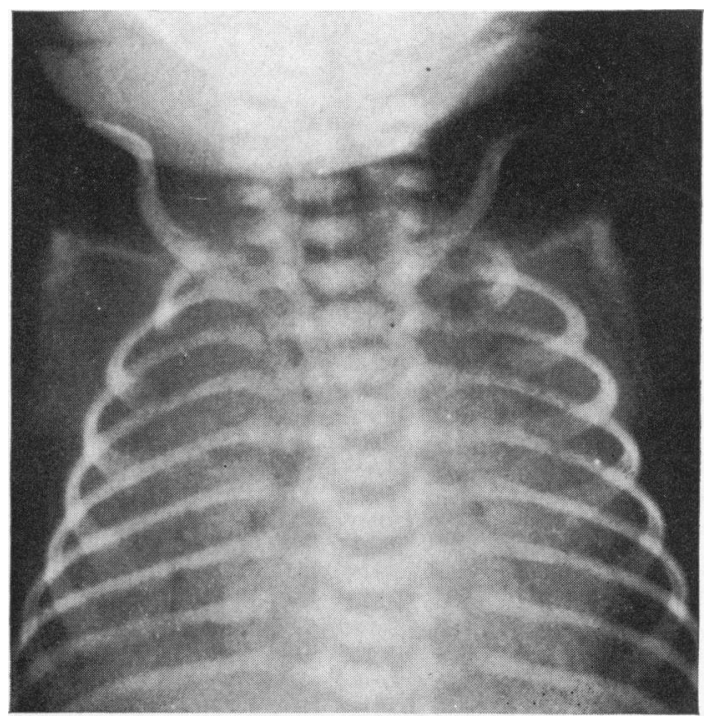

FIG. 7.-Position of the endotracheal tube checked by $\mathrm{x}$-ray (case of RDS which survived after 10 days on $I P P V)$. 
tor's left hand, the laryngoscope, and the baby's head and jaw should now form one united assembly.

The naso-tracheal tube, already fixed to the modified connector, is passed down a nostril until it is in view in the oropharynx. The tip of the tube is passed about $1 \mathrm{~cm}$. into the oesophagus. The shaft is now grasped by a pair of small Magill's forceps held in the right hand and the tube is withdrawn from the oesophagus. This manœuvre gives an adequate length of tube beyond the forceps. The soft palate is now displaced somewhat by the forceps. The tip of the tube is guided to the larynx, and then by manipulation directed downwards and backwards to follow the direction of the trachea. The condition of a baby in respiratory failure may well have deteriorated by the time intubation is complete, and manual IPPV via the naso-tracheal tube should be temporarily administered, using $100 \%$ oxygen.

Every endeavour is made to maintain sterility; sterile gloves are worn and intubation instruments are sterilized.

\section{T-piece Suction Connector}

This piece of equipment is illustrated in the top left-hand corner of Fig. 8, and is part of the new Oxford pattern endotracheal tube connector, and fits on to the male mount of the endotracheal tube connector. When undertaking tracheal toilet it is more convenient to detach the two connectors completely as has been done in Fig. 4. When the baby with its indwelling endotracheal tube connector is to be attached to the suction connector, which in turn connects with the tubing of the ventilator, it is important to take the baby to the suction connector-not to tense the ventilator tubing in order to take the suction connector to the baby, which will tend to cause the union to slip. With proper positioning of sandbags, as shown in Fig. 5, not only will the two connectors not separate but artificial ventilation will continue in spite of a loose union.

\section{Tracheal Suction Catheter}

The sterile disposable Portsmouth pattern

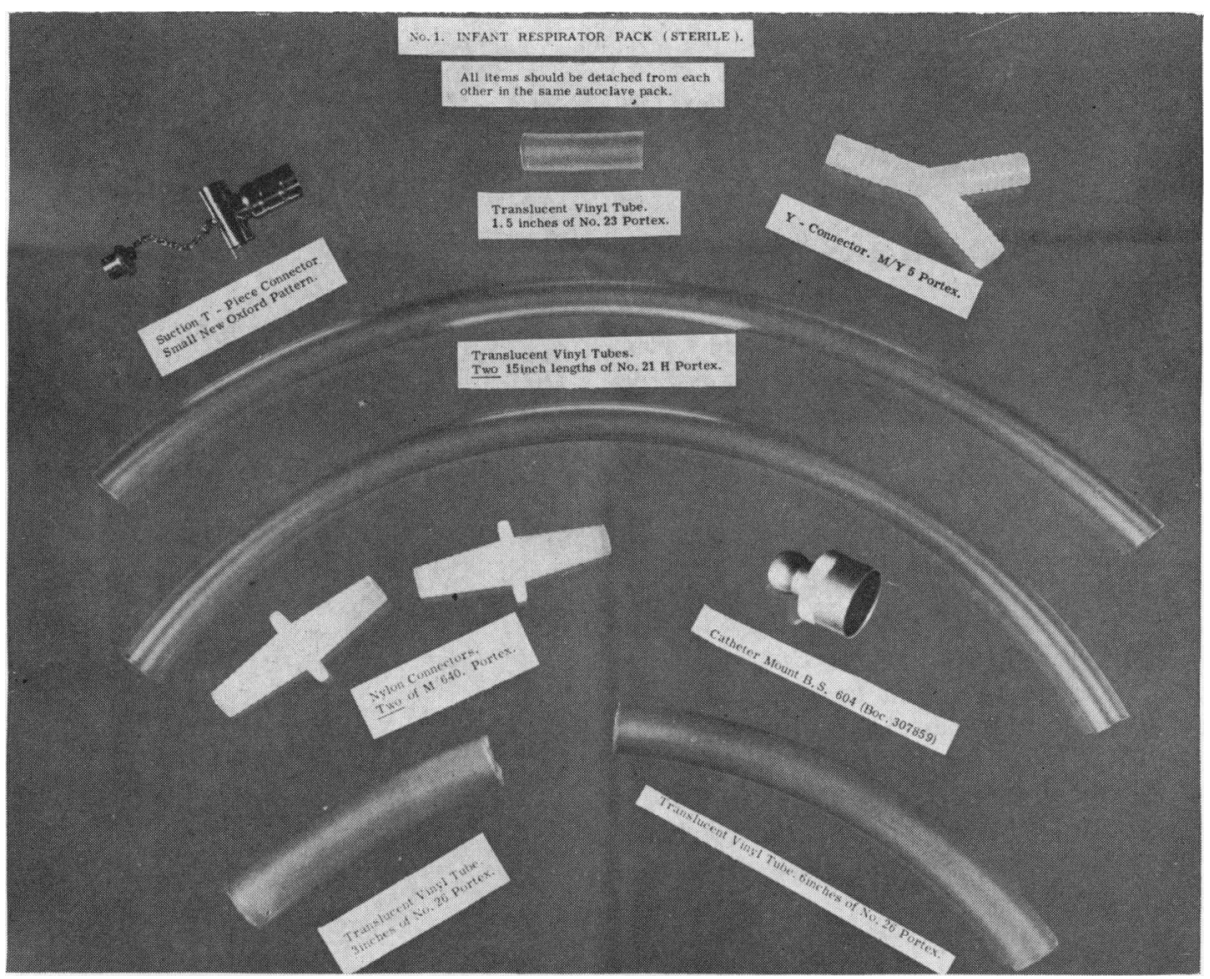

FIG. 8. - The components of the sterile infant respirator pack (Catheter mount B.S. 604, BOC 307859 made by British Oxygen Co., Great West Road, Brentford, Middlesex; sources of other components listed in text). 
catheter ${ }^{\star}$ has proved satisfactory (Tunstall and Hodges, 1961). There is a lateral aperture adjacent to the distal open end. This is designed to reduce the suction biopsy effect on the mucous membrane which occurs when suction is applied via a single aperture. Nurses use the size No. 5 Charrière gauge suction catheter for routine tracheal aspiration. Only medical staff pass the No. 6 catheter, as this size virtually fills the lumen of the $2.5 \mathrm{~mm}$. internal diameter endotracheal tube.

The larger catheter is passed to remove any inspissated secretions which may line the lumen of the endotracheal tube at its distal end. Such a lining has been observed from time to time. It forms an annulus through which the No. 5 suction catheter passes, but nevertheless it is a restriction of the airway and needs to be removed.

Sterile technique during tracheal toilet is maintained by the operator using sterile gloves. During the procedure the suction catheters need to be cleared frequently by sucking sterile water.

The last manœuvre the nurse performs after clearing the trachea of secretions is to grasp the suction catheter adjacent to the metal connector immediately before withdrawal, and after withdrawal, to compare the depth of insertion with a minimum length marked out by the medical attendant. This ensures that she has aspirated beyond the tip of the endotracheal tube.

In most of the premature babies that we have ventilated, secretions have not been excessive and 2-hourly aspiration has been satisfactory.

It is important to have the suction turned on during insertion of the catheter, as this helps to prevent distal impaction of secretions, and also helps to reduce contamination of the catheter by gravitational regurgitation from the suction apparatus tubing. The babies are given three positive pressure 'sighs' of $50 \mathrm{~cm}$. $\mathrm{H}_{2} \mathrm{O}$ pressure every hour, and after each tracheal aspiration.

\section{Pharyngeal Secretions}

These secretions are not routinely aspirated. The babies are nursed on their sides and pharyngeal secretions are either swallowed or drained externally by gravity. Naso-gastric tubes are not favoured in very ill babies as they promote regurgitation. A negative ventilatory phase is not used as it would be liable to cause aspiration of pharyngeal secretions alongside loose-fitting endotracheal tubes.

\section{Feeding}

Gastrostomy is normally employed in babies who

* William Warne and Co., Barking, Essex. are clearly going to be on IPPV for more than 24 hours (Jones and Reid, 1966). Gastrostomy facilitates the feeding of ill neonates without disturbance. There is every advantage in early feeding (Stoneman, 1967).

\section{Variable Dead Space}

Fig. 5 portrays a large ventilatory apparatus dead space caused by the length of the tubing from the $\mathrm{Y}$-junction of the inspiratory and expiratory tubes to the T-piece suction connector. This baby had suffered recurrent attacks of apnoea and was being treated by IPPV. His arterial $\mathrm{PCO}_{2}$ was 33 $\mathrm{mm} . \mathrm{Hg}$ at the time the photograph was taken; he is now alive and well. It is our policy not to ventilate babies at less than $20 \mathrm{~cm} . \mathrm{H}_{2} \mathrm{O}$ pressure, in order to prevent progressive pulmonary collapse, so that if a baby has fairly normal lungs, in order to prevent hypocapnia, we increase apparatus dead space rather than reduce ventilatory rate or ventilatory pressure. It is appreciated that a leak of gas past a loose-fitting endotracheal tube is a factor in $\mathrm{CO}_{2}$ wash-out. In cases such as respiratory distress syndrome (RDS) dead space needs to be at a minimum.

In some cases, difficulty was experienced in weaning off the ventilator in the presence of a low $\mathrm{P}_{\mathrm{a}} \mathrm{CO}_{2}$, but correction of the gas tension overcame the difficulty.

\section{Distal Ventilator Tubing}

The concept of distal and proximal ventilator tubing has arisen from the technique of sterilization of equipment which has been adopted. All ventilator equipment which enters the baby's incubator (i.e. beyond unions $A_{1} / B_{1}$ and $A_{2} / B_{2}$ in Fig. 9) is sterilized by autoclaving. These items can be packaged together for sterilization (Fig. 8).

The holes of entry into the incubator for the inspiratory and expiratory tubes are made a little below the level of the tray on which the baby lies (Fig. 5), in order to promote gravitational drainage of condensed water in the tubes.

One advantage of unions at $B_{1}$ and $B_{2}$ (Fig. 9) is that should a baby require transport in the incubator to or from the operating theatre, one limb of the tubing can be attached to oxygen and the other limb to an open-ended $500 \mathrm{ml}$. anaesthetic rebreathing bag. The attendant can then perform manual IPPV during transport using the now standard $T$-piece paediatric anaesthetic circuit (Rees, 1963).

\section{Dummy Lung}

The dummy lung (Fig. 9 and 10) consists of a $500 \mathrm{ml}$. double-ended antistatic rubber rebreathing 


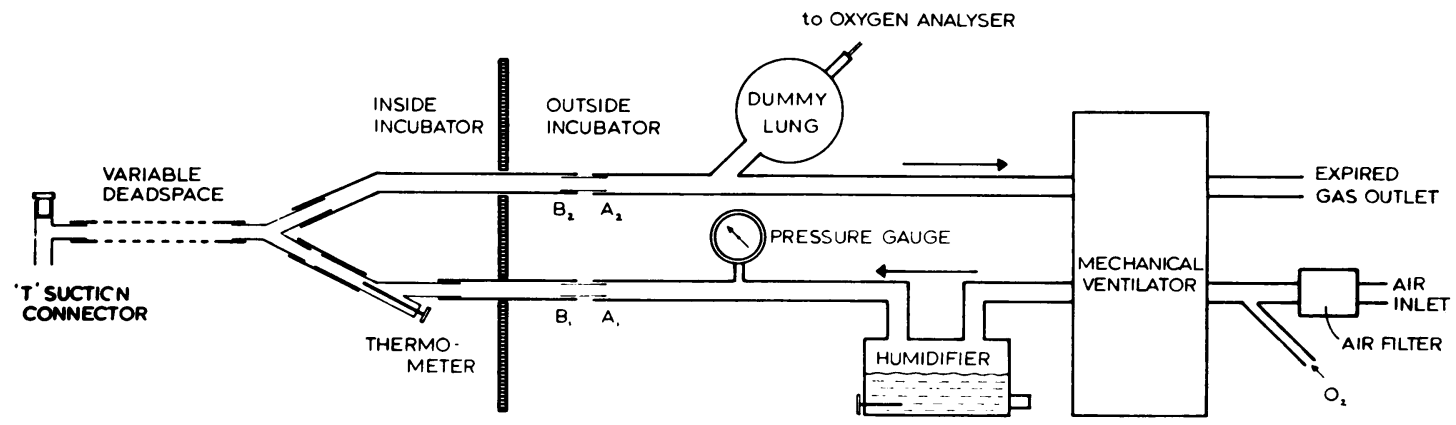

FIG. 9.-Mechanical ventilator circuit for neonates.

bag. This bag at zero gauge pressure tends to collapse flat, due to the combined effects of its manufactured moulding and of gravity. When its static compliance is studied, gauge pressure inside the bag does not begin to rise until 400 to $500 \mathrm{ml}$. gas have been accommodated. The size and compliance of the rubber bag are such that it is possible to achieve any desired pressure in the circuit of the ventilator that we use. Should this not have been the case, the rubber bag would have to have been placed inside a bag of nylon net in order to limit its expansion to a volume of 400 to $500 \mathrm{ml}$.

The dummy lung functions as a volume-limited circuit leak. It increases the minute volume of the ventilator circuit. This has the following advantages. (1) It enables standard medical gas flow meters to be used for selecting the mixture of air and oxygen required in the circuit; (2) it makes the use of many currently available ventilators practical for neonates, thus eliminating the need for a special neonatal ventilator; and (3) it reduces the higher temperature gradient that would otherwise be necessary between the hot water humidifier and the baby's trachea.

Instead of a dummy lung, Bloch (1967) recommends a simple controlled leak in the ventilator circuit for neonates; this, however, is of no help to the baby during out-of-phase breathing.

We find that all neonates breathe out-of-phase with the ventilator for variable periods of time, usually when being handled or in respiratory failure. Arterial $\mathrm{Po}_{2}$ does not rise when ventilation has been controlled with the help of relaxant drugs in a baby

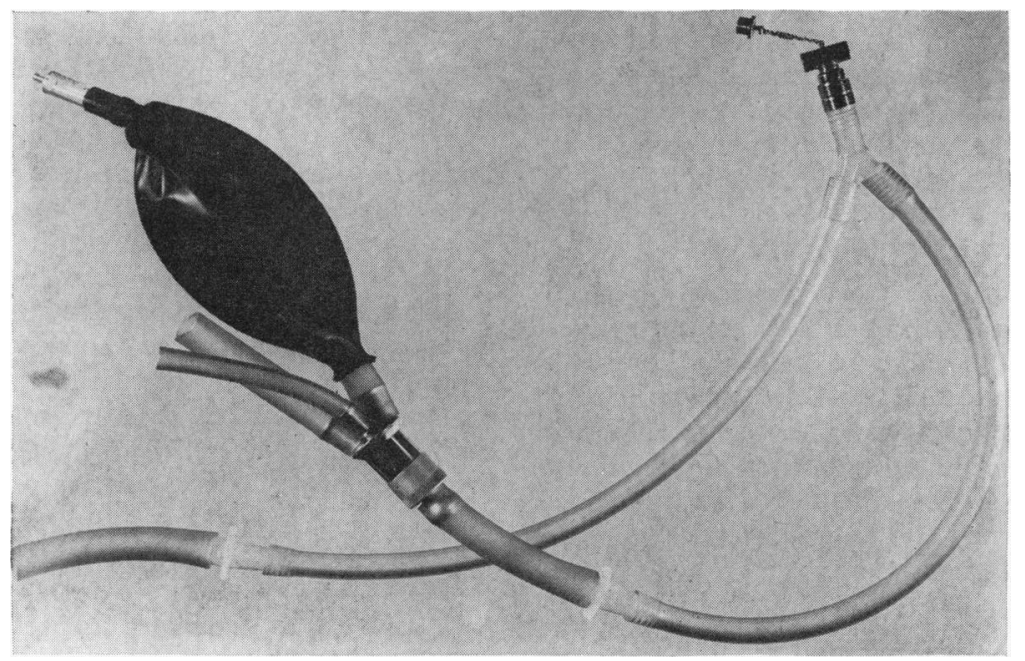

FIG. 10.-The 'dummy lung'. In this picture the small diameter tube leading to a pressure gauge, not shown, is on the same limb (expiratory) as the dummy lung. 


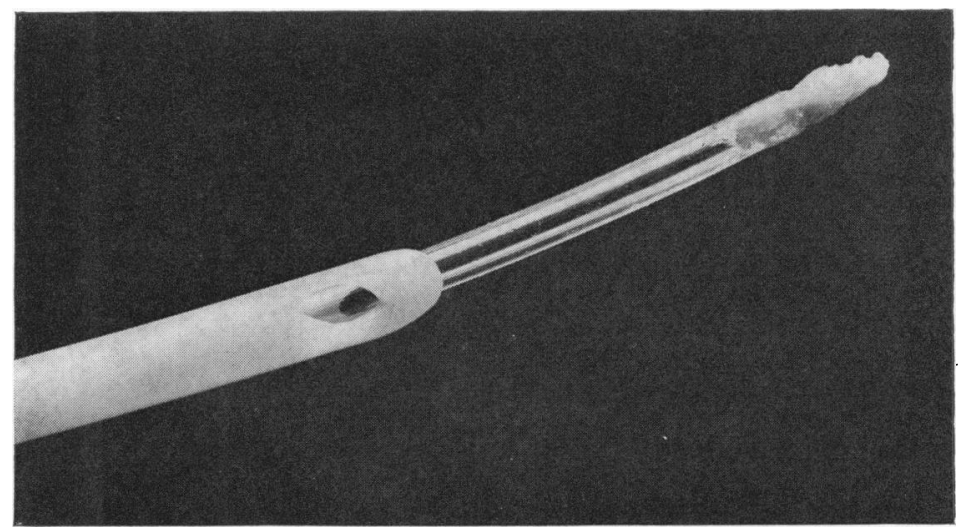

Fig. 11.-Accidental failure to humidify. Inspissated secretions which had obstructed tracheal tube, and had been cleared by the suction tube.

previously breathing out-of-phase with the ventilator. The dummy lung outlined above probably reduces the work of out-of-phase breathing.

The use of the dummy lung is simplest with ventilators that are time-cycled pressure generators. The problems of automatic ventilation in infants and children are clearly set out by Mushin, Mapleson, and Lunn (1962).

\section{Humidification}

The delivery to the trachea of gases that are nearly saturated with water vapour at body temperature is essential, and is simply achieved by using a hot water humidifier in the inspiratory line. The temperature on the humidifier, usually about $50-60^{\circ} \mathrm{C}$., is adjusted so that the gas temperature near the dead space tubing is in the range of $33-36^{\circ}$ C. This method of humidification has been found to be adequate for the neonates that have been treated so far.

Fig. 11 illustrates what occurred when a baby was disconnected from the ventilator (the naso-tracheal tube being left in situ) for a trial period and the medical attendant omitted to turn on the cold aerosol nebulizer to the incubator. The humidification ordinarily obtainable in incubators is not adequate for artificial airways, and so the secretions crusted in the tube. (The baby's life was saved.)

\section{Sterilization}

All ventilator tubing distal to unions $A_{1} / B_{1}$ and $A_{2} / B_{2}$ (Fig. 9) is sterilized by autoclaving in a single pack (Fig. 8).

Sterilization proximal to unions $A_{1} / B_{1}$ and $A_{2} /$ $B_{2}$ is by the circulation of formaldehyde vapour (Sykes, 1964); $A_{1}$ and $A_{2}$ are joined together. The expired gas outlet is joined to the air inlet port, the negative bellows are turned on, and then there is a completely closed circuit. The formaldehyde is placed in the hot water humidifier. Recirculation is for 4 hours. Deformalinization is obtained either by flushing for 24 hours without recirculation, or by recirculating ammonia vapour for 30 minutes and a subsequent flushing without recirculation for 30 minutes. For flushing, the expired gas outlet and air inlet should be disconnected and the humidifier water changed. It is important that (1) water in the humidifiers should always be sterile and distilled; (2) emptying or filling humidifiers should be with sterile syphon tubes or funnels; and (3) humidifiers should be kept empty during periods out of service. This will prevent colonization by pyocyaneus. After sterilization, the outside of the ventilator is swabbed with $0.5 \%$ chlorhexidine in $70 \%$ industrial methylated spirit, and is then covered with a dust sheet. Before use the ventilator should be turned on for 5 minutes and then checked for traces of formaldehyde vapour.

\section{Ventilator}

We tend to agree with the following quotation from the Lancet (1965): 'The tedious argument about the virtues of respirators not invented over those readily available can be ended, now that it is abundantly clear that success of such apparatus depends on the skill with which it is used.'

Ventilators 'not invented' are often designed on the following assumptions. (1) Apparatus dead space should be minimal in all cases; (2) ventilator tubing should be of narrower bore for children than for adults; (3) tidal and minute volumes should be forecast, pre-set, and fixed; and (4) apparatus needs to be small in bulk. 
These four points are answered as follows. (1) Under the heading of 'variable dead space' as above; (2) when babies are ventilated with reference to ventilator rate and pressure and monitored by blood gas measurements, the diameter of the inspiratory and expiratory ventilator tubing is unimportant; narrow bore tubing increases the danger of discrete columns of condensed water in the inspiratory tube being projected into the trachea; (3) a close fit of the tube in the subglottic region is presupposed, and if this is aimed at in every case, the problems of subglottic oedema, possibly followed by tracheostomy, will arise; (4) the baby is already in an incubator which is of necessity bulky. The only ventilator apparatus inside the incubator is the twin inspiratory and expiratory tubing. The size of a ventilator which stands adjacent to the incubator is hardly relevant.

Babies are more conveniently ventilated with reference to pressure and rate. If the pressure source has a large reserve of volume and flow, then minor gas leaks from the system, for example, due to a loose-fitting endotracheal tube or a partially slipped connexion, are not important. When the reference points of IPPV are rate, pressure, and apparatus dead space, any or all three are adjusted to produce desired blood gas tensions. The measurement of tidal and minute volumes, which is difficult in babies, then becomes irrelevant as far as management is concerned. This, combined with the use of the dummy lung, means that certain existing ventilators, which are used for adults and available on the market, are satisfactory for neonates. The East-Radcliffe respirator PNA $1^{\star}$ is used in Aberdeen. It is a time-cycled pressure generator.

\section{Charting}

The ventilator chart as illustrated in Fig. 12 is a facsimile of one being used for a baby on a ventilator, and is largely self-explanatory. It serves two purposes; first, it records the condition of the baby; and second, it is a check list for the nurse so that looking after a baby on a ventilator becomes a simple routine.

The following points may need to be explained. Peripheral circulation is assessed by capillary return following compression of the nail bed. 'Vent. resp. movt.' means the movement of the chest that occurs in phase with the ventilator. 'Dry oxygen input' refers to the oxygen being fed to the incubator. It is intended to help the infant who makes spontaneous ventilatory effort when temporarily disconnected from the ventilator.

\footnotetext{
* H. G. East and Co. Ltd., Cowley, Oxford.
}

On the back of the ventilator chart are instructions (Fig. 13).

\section{Notices}

Two notices (Fig. 6, and the following) are normally on display in the same room as the baby on a ventilator.

\section{EMERGENCY ORDERS FOR INFANTS ON MECHANICAL ARTIFICIAL VENTILATION}

\section{SECTION A:}

Failure of mechanical ventilation-

I. Send for help.

2. Disconnect infant from ventilator. LEAVE THE INFANT'S TRACHEAL TUBE AND CONNECTOR IN PLACE.

3. If the infant is breathing on own (stimulate if necessary), flush (incubator) with oxygen as demonstrated. Listen to airway sounds over the mouth of the tube and connector with a stethoscope. Check that the infant retains a good colour.

4. If the infant is not breathing or cannot maintain a good colour, apply IPPV by hand as demonstrated 30-40 times per minute.

\section{SECTION B:}

Blockage or displacement of tracheal tube.

If the tube is blocked, diagnosed (a) in infants trying to breathe by absence of airway sounds over the mouth of the tube and connector when listening with a stethoscope, (b) in infants not breathing by failure of ventilation movements of chest and abdomen when IPPV is applied1. Send for help.

2. Make sure tube is not kinked by abnormal position of connector at the nose.

3. Try to clear tube with a suction catheter.

4. If not cleared by above, remove tube (this is a last resort).

5. Flush (incubator) with oxygen as demonstrated.

6. Apply IPPV by face mask, if necessary, until doctor is able to perform emergency intubation.

\section{Extubation}

Extubation takes place when it is decided to try to wean the baby off the ventilator. The babies in respiratory failure due to RDS pose a special problem as they are usually weaned while there is still some respiratory failure present. They are therefore not usually tried off the ventilator with the naso-tracheal tube in situ, because of the extra work of breathing occasioned by the relatively narrow diameter of the tube.

The decision to wean off the ventilator can only be taken on the spot, but in general if the $\mathrm{P}_{\mathrm{a}} \mathrm{CO}_{2}$ is not above $60 \mathrm{~mm}$. $\mathrm{Hg}$, if the baby does not collapse or become a poor colour when being handled or having his trachea aspirated, if he is tolerating his feeds, and if he is not developing a base deficit while on $40 \%$ oxygen, he is tried off the ventilator.

Immediately before extubation, the trachea, pharynx, and unoccupied nostril must be aspirated using separate catheters for each. Extubation is 


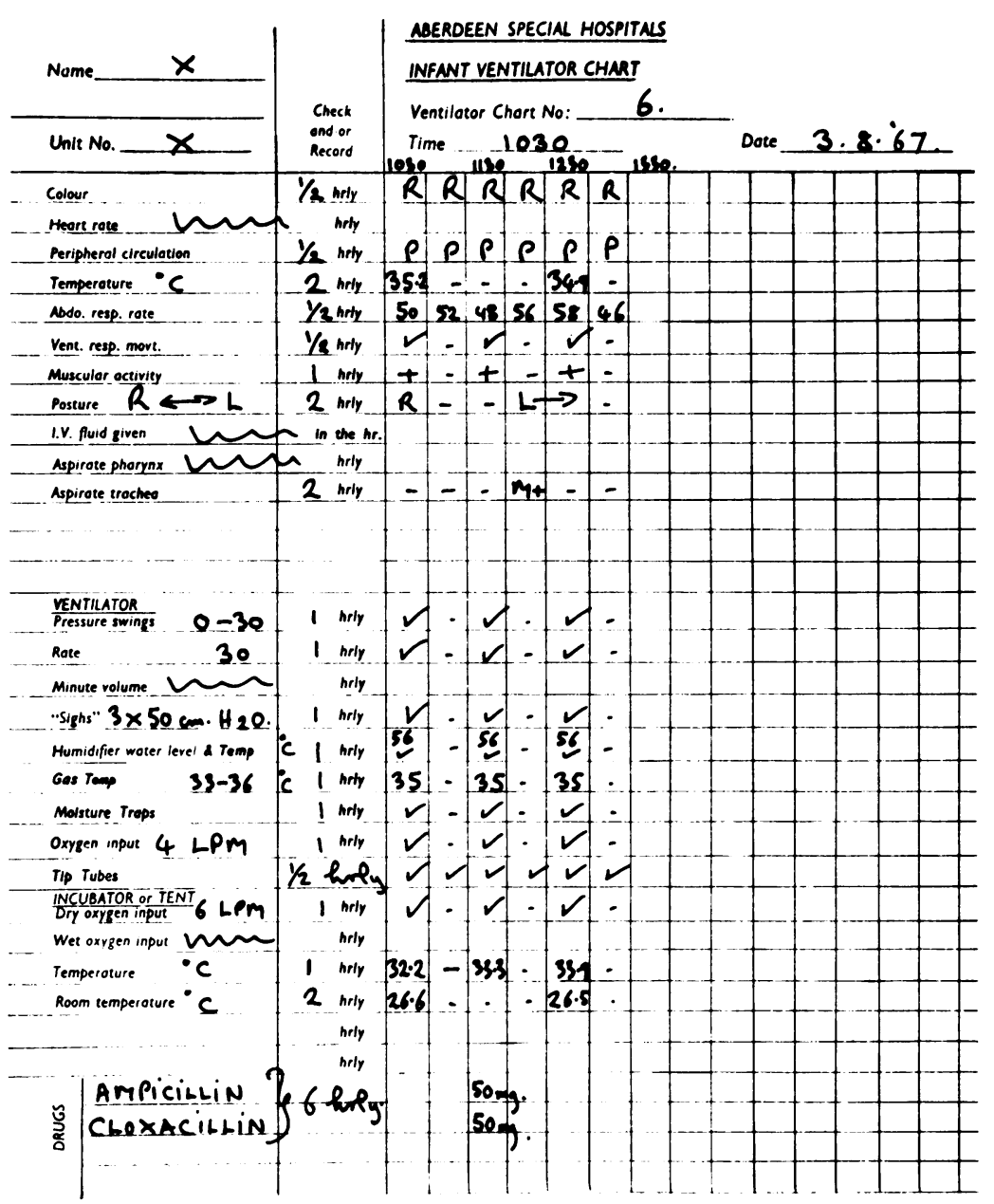

FIG. 12.-The ventilator chart.

rapidly performed while a positive pressure of 50 $\mathrm{cm} . \mathrm{H}_{2} \mathrm{O}$ is being exerted. This causes a 'cough' to follow the tube, and secretions around the tube are generally expelled out of the trachea. The pharyn $x$ is then again aspirated. The nasal passage in which the tube has been residing is usually full of mucoid material and must be aspirated forthwith as neonates always attempt to breathe through their noses.

During extubation, the incubator should be flushed for a few minutes with oxygen. After extubation, the cold water nebulizer should be turned on for two hours. The oxygen concentration in the incubator should be that which maintains the baby in a satisfactory colour. The baby should in no way be handled for at least 2 hours after extubation. The decision to re-intubate is taken either on appearance or on an episode of collapse or, when the decision is more difficult, on serial blood gas estimations over several hours.

\section{Blood Volume}

It has been observed from time to time that babies with RDS, with or without oedema, sometimes appear to improve while on IPPV when given a transfusion of $20-30 \mathrm{ml}$. blood.

\section{Inspired Oxygen Concentration}

High inspired oxygen concentrations for short periods of time are permissible to help treat episodes of collapse or periods of cyanosis. It is probable 
(1) The venplitotor hos been set os follows: Pressure 0-30, Rate 30.

Added saggem 4 LPM $\equiv 54 \%$ Insp.02. Come.

N.B. O foll in the see pressure usually meons o disconnecevon or leok.

(x) Pasture posient $R \leftrightarrow L$ side $2 \mathrm{hul}_{y}$.

(3) The trocheal suction catheter is to be used only for the trocheo. The minimum
depeh of insertion of the trocheol suction cotheter is to be ass.

(1) Perform "SIGH" ofter eoch trocheol asplrotion ond ot the following times

(5) introvenous fluids $\mathrm{Ni}$

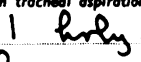

(6) Feeds Gastrostony regime.

(7) CHART CODES (more thon one letter may be needed for each entry)

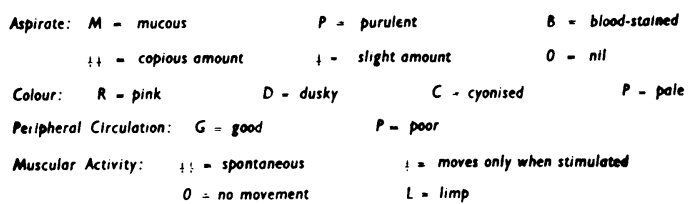

(8) Residen: : Please Arrange.-

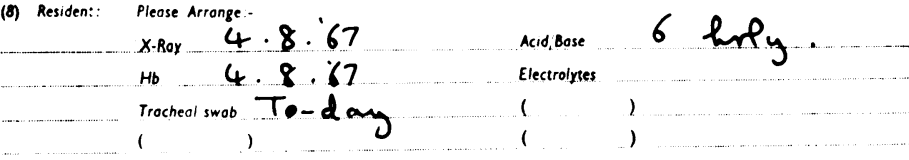

FIG. 13.-The reverse aspect of the infant ventilator chart (Fig. 12).

that the administration of inspired oxygen concentrations of over $50 \%$ for more than 6 hours is damaging to alveoli. In RDS, high oxygen concentrations are of little benefit if the right-toleft shunt is severe. For such cases we shall have to await the advent of the 'artificial placenta'.

\section{Ventilatory Pressures}

As has been stated, all our infants are subjected to 'sighs' of $50 \mathrm{~cm} . \mathrm{H}_{2} \mathrm{O}$ pressure at hourly intervals. Those infants with RDS who require as much as $50 \mathrm{~cm} . \mathrm{H}_{2} \mathrm{O}$ pressure for more than a few hours to maintain an improved, but not correct, blood gas picture usually die. We have no good reason to change our policy of keeping to a minimum inflation pressure of $20 \mathrm{~cm} . \mathrm{H}_{2} \mathrm{O}$.

\section{Ventilatory Rates}

The uppermost ventilatory rates of the machine we use are 37 and 30 cycles per minute. We usually use 37 c.p.m. for cases in respiratory failure and 30 c.p.m. in cases such as apnoeic attacks, and are satisfied with these rates.

\section{Nursing}

There is a trained nurse continuously present with the infant in the ventilator room. She is able to look after 2 ventilator cases simultaneously. We have had as many as 4 infants on ventilators simultaneously.

\section{Discussion}

The method described has been in use in Aberdeen for several years (Reid and Tunstall, 1966; 
Reid and Mitchell, 1966; Reid, Tunstall, and Mitchell, 1967), and to date we have treated over 90 newborn infants by prolonged IPPV in the Special Nursery of the Aberdeen Maternity Hospital. We have similarly treated a further 41 infants in the Royal Aberdeen Hospital for Sick Children.

Paediatricians elsewhere have reported difficulty in stopping IPPV once it has been started: thus, Tizard (1967) states that 'rarely does one put a baby with classical respiratory distress on a respirator and ever get it off'. We have not experienced such difficulties.

Complications of IPPV which have been recorded include laryngeal damage, subglottic stenosis, pulmonary collapse, and the 'respirator lung syndrome' (Lancet, 1967). Fearon (1966) reported that 27 children out of 72 intubated for more than 8 hours suffered laryngeal damage, while 6 later developed severe subglottic stenosis. Hardcastle (1967) reported serious complications. We have had no case of subglottic oedema or stenosis, even though many infants have had naso-tracheal intubation for prolonged periods. Freedom from these and other respiratory complications of ventilation is attributable to scrupulous attention to detail as outlined.

\section{Summary and Conclusions}

A detailed account of the technique of prolonged artificial ventilation in the newborn infant is given. The technique described may be successfully managed by paediatric and nursing staff in the course of their normal duties.

\section{REFERENCES}

Bloch, E. C. (1967). Modifying the Bird Mark 8 ventilator. Anaesthesia, 22, 356.

Fearon, B. (1966). Quoted by Hardcastle (1967).

Hardcastle, B. (1967). Prolonged intubation and subglottic stenosis. Brit. med. F., 1, 112.

Jones, P. F., and Reid, D. H. S. (1966). Gastrostomy in neonatal respiratory failure. Lancet, 2, 573.

Lancet (1965). Respirator treatment in hyaline-membrane disease. 2, 1227 .

- (1967). Pulmonary respirator syndrome. 1, 992.

Mushin, W. W., Mapleson, W. W., and Lunn, J. N. (1962). Problems of automatic ventilation in infants and children. Brit. $\mathcal{F}$. Anaesth., 34, 514.

Rees, G. J. J. (1963). Management of neonatal asphyxia. In The Obstetrician, Anaesthetist and the Paediatrician in the Management of Obstttric Problems, p. 125. Ed. by T. Barnett and J. J. Foley. Pergamon Press, Oxford.

Reid, D. H. S., and Tunstall, M. E. (1966). The respiratory distress syndrome of the newborn. Anaesthesia, 21, 72.

- and Mitchell, R. G. (1966). Recurrent neonatal apnoea. Lancet, 1, 786.

_-, Tunstall, M. E., and Mitchell, R. G. (1967). A controlled trial of artificial respiration in the respiratory-distress syndrome of the newborn. ibid., 1, 532.

Stoneman, M. E. R. (1967). Hyaline membrane disease. Brit. med. F., 1, 298.

Sykes, M. K. (1964). Sterilizing mechanical ventilators. ibid., 1,561 .

Tizard, J. P. M. (1967). Respiratory problems in the neonate. Hosp. Med., 1, 521.

Tunstall, M. E., and Hodges, R. J. H. (1961). A sterile disposable neonatal tracheal tube. Lancet, $1,146$. 